\section{Phantoms in medicine: The case of ophthalmology}

Carmelo De Maria,1,2 Sara Iorio, ${ }^{1}$

Francesca Montemurro, 1

Gabriele Maria Fortunato,1,2

Michela Ori, ${ }^{3}$ Vittoria Raffa, ${ }^{3}$

Giovanni Vozzi1,2

1E. Piaggio Research Center, University of Pisa; 2Department of Information

Engineering, University of Pisa;

3Department of Biology, University of

Pisa, Pisa, Italy

\begin{abstract}
Physical and in-silico phantoms have revealed extremely useful in the development of new surgical techniques and medical devices and for training purposes. The fabrication of eye phantoms requires knowledge of anatomy and physical principles beyond the eye physiology and medical instruments used in the clinical scenario. After a proper definition of phantoms and the discussion about their classification, the present work reviews the various phantoms developed in ophthalmology, illustrating the rationale of their design.
\end{abstract}

\section{Introduction}

\section{Use of phantom in medicine}

The great progress in medicine over the last 40 years is strongly linked to the increasingly important use of technological tools, both for diagnostic and therapeutic purposes. This has led to an increased accuracy of a diagnosis, moving from the subjectivity of the anamnesis and the sensibility of physicians, to the objectivity of quantitative measurements obtained, for example, by computed tomography or magnetic resonance imaging. Consequently, this has resulted in an improvement in the therapy that, nowadays, can provide targeted solutions on the individual patient as well as take advantage of minimally invasive surgical instruments.

In the development of new medical devices, the final goal is to obtain a measure or provide a treatment, while ensuring the accuracy in the result and maximum protection both for the patient and the healthcare provider. As an example, after the discovery of X-rays, it became evident the benefits that these could bring to the medicine, synthesized by the radiography of the hand of Prof. W. Roentgen's wife. 1 Nevertheless, the harmful effects of high doses of radiations were so visible (erythema and skin desquamation) that it was not easy, besides unethical, to find volunteers willing to expose themselves to radiation doses for the testing new devices. To respond to this shortage of volunteers, phantoms, i.e. simulators of patients, were invented.

From a bioengineering point of view, $a$ phantom can be defined as an object made to imitate the human figure, or a part of it, using non-living materials to simulate a particular physical or chemical property. Phantoms are mainly used for the development, safety checking, calibration and training in the use of diagnostic, therapeutic or with other purposes devices, which come in contact with human beings.

The phantom thus constitutes an immediate testing bench for a technology, ensuring repeatability and reproducibility, as well as the comparison between different instruments.

Moreover, overcoming any ethical problem, phantoms find large use in surgical training, bringing considerable advantages regarding the patient safety, management of operating rooms, and the training itself. In this context, compared to the use of anatomical specimens coming from corpses, phantoms generally guarantee lower costs, higher security, and more controllable physical/chemical properties. ${ }^{2,3}$ As example, the elasticity of soft tissues in corpse tissues is frequently altered by treatments with formaldehyde, or other properties, such as the viscosity of the vitreous humor, widely change shortly after death.

As an indirect but equally important consequence, the use of phantoms reduces the need of cavies, in accordance with the principles of Replacement, Reduction and Refinement ( $3 R$ rules) on the animal testing. ${ }^{4}$ Of course, as for all models of a physical system as complex as the human body, the accuracy of the information that can be obtained by simulating an operation on a phantom is limited and strictly connected both to the knowledge of the system to be emulated and to the structural characteristics of the phantom itself. Nevertheless, to date, phantoms, either physical or virtual, constitute an essential instrument for the development of new technologies in medicine.

\section{Classification}

Given the heterogeneity of the human body tissues and the great variety of situations of interest (from the radiations of mobile phones to the scalpel of a surgeon), the design and composition of a phantom will change according to the purpose for which it is constructed, which will deter-
Correspondence: Carmelo De Maria, E. Piaggio Research Center, University of Pisa, Largo L. Lazzarino 1, 56122 Pisa, Italy. Tel.: +39.050.2217073.

E-mail: carmelo.demaria@unipi.it

Key words: Ocular phantom; Phantom classification; Imaging; Dosimetry; Surgical training; Pharmacokinetics.

Contributions: the authors contributed equally.

Conflict of interest: the authors declare no potential conflict of interest.

Funding: this research received funding from University of Pisa Fondi PRA 2016 programme.

Received for publication: 12 October 2018.

Revision received: 2 April 2019

Accepted for publication: 30 April 2019.

This work is licensed under a Creative Commons Attribution NonCommercial 4.0 License (CC BY-NC 4.0).

CCopyright: the Author(s), 2019

Licensee PAGEPress, Italy

Biomedical Science and Engineering 2019; 3:61 doi:10.4081/bse.2019.61

mine its physical characteristics, such as shape and size. For instance, if the interest is directed to optical or mechanical properties, an identical part of the body can be emulated by different materials.

Besides the immediate classification based on the anatomical district to be simulated (tissue, organ, or body part), a general first subdivision among the various phantoms, commercially available and/or described in literature, can be based on the geometry they present, obtaining:

Non-anthropomorphic phantoms, in which only the physical and chemical properties of the tissue of interest, and its gross sizes (such as thickness), are reproduced, but not the overall shape of an organ; e.g. a cylindrical container filled with an appropriate material can be used to simulate the torso of a person;

Anthropomorphic phantoms, which reproduce properties and shape of organs and tissues. Nowadays, thanks to the development of additive manufacturing technologies, coupled with tomographic scans of the human body, accurate three-dimensional physical models can be created at a relatively low cost. In parallel, the increased capacity of computers in handling large files allows the construction of extremely realistic 
virtual models of the human body.

Focusing on constructive methods, it is possible to classify phantoms in:

Physical phantoms, i.e. models of the human body on which perform tests and measurements using directly medical instruments. This category can be further divided on the basis of the physical state of the materials used, obtaining: i) Solid phantoms, typically composed of resins or hydrogels, to which a more or less stylized shape can be given; ii) Liquid phantoms, in which solutions, typically water-based, reproduce the features of interest. They require a container which can be anthropomorphic;

- Virtual (or in silico) phantoms, i.e. computational models of the human body, on which it is possible to act through the peripherals of the computer itself. This category includes: i) Interactive phantoms, also classifiable as serious game, characterized by the behaviors of the model identified a priori and used in the surgical training; ii) Phantoms for calculation, primarily used in research and development, for the simulation and estimation of quantities hardly measurable in vivo. They can be further distinguished in: ii.i) Probabilistic, based on Monte Carlo simulation methods, mainly used in dosimetry issues; ii.ii) Deterministic, based on numerical solution of partial differential equations (finite elements, finite differences, finite volumes), which find application, for example, in simulations of the mechanical behavior of a tissue subjected to particular loads.

These two classifications can be combined in a double entry table leading to a subdivision of phantoms based on their structural features. Some real examples are: cylinders for dosimetry measurements (physical non-anthropomorphic), manikins for catheterization and laparoscopic surgery (physical anthropomorphic), models with a spherical geometry used to validate the numerical solution with an analytical one (virtual non-anthropomorphic) and computer-assisted surgery simulators (virtual anthropomorphic).

Besides their constructive aspects, it is extremely useful to classify phantoms in function of their application, as shown in Figure 1, described below:

Phantom for imaging: used in the design, calibration, testing and accreditation of the imaging equipment (ultrasound, optical tomography, etc.), paying particular attention on parameters as resolution and accuracy of the final image; 5

- Phantom for dosimetry: used to calcu- late the radiation dose absorbed by a person, with diagnostic purposes, for treatment and prevention (radioprotection); 6,7

Phantom for training: used for the validation of new surgical instruments, as well as for training of surgeons (e.g., for vitrectomy operations); $;, 9$

Phantom for pharmacokinetics: useful for estimating the distribution of biomolecules of interest (e.g., drugs, oxygen) in specific areas of the body.

It is important to note that in all these fields either physical or virtual phantom, both non-anthropomorphic or anthropomorphic ones, can be used.

Based on the latter classification, all the mentioned above aspects, from the applications of phantoms to their construction techniques and design methodologies, will be described in the following sections, focusing on the phantoms used in ophthalmology.

\section{The human eye}

\section{Anatomy and function}

The eye is the main organ of the sight apparatus. Its particular organization and its connections with the brain allow it to transmit to the nervous system a set of signals which reproduce the images of the external world. This process involves a complex dioptric system consisting of the cornea, crystalline lens, aqueous humor and vitreous body.

The eye presents an approximately spherical shape, with a weight of about 7-9 g. Its wall consists of three superimposed membranes, called tunics: i) external or fibrous, composed of the sclera and cornea; ii) media or vascular, also known as uvea, formed by iris, ciliary body and choroid; iii) internal or nervous, constituted exclusively by the retina.

The sclera occupies the $93 \%$ of the surface of the eye, whereas the remaining part is occupied by the cornea. Consisting mostly of connective tissue, the sclera, which has a radius of $12.7 \mathrm{~mm}$ and a thickness of about $1 \mathrm{~mm}$, has a mainly structural and protective functions. Its external surface presents several orifices, the largest of which is the exit point of the optic nerve. The cornea instead has a radius of curvature of about $8 \mathrm{~mm}$ (hence the roughly spherical shape of the eye). It is a transparent structure without blood vessels and, therefore, it is nourished by the sclera. Regarding the uvea, the iris constitutes its anterior part; it determines the eye color and presents a central hole, the pupil, whose amplitude varies from 2 to $8 \mathrm{~mm}$. The ciliary body plays a key role in the vision due to the presence of the ciliary muscles, which determine the variation of the radius of curvature of the crystalline lens (accommodation). The choroid has a variable thickness, which ranges from $0.1 \mathrm{~mm}$ anteriorly to $0.2 \mathrm{~mm}$ posteriorly; it is the rear part of the uvea and represents the eye vascular membrane, providing adequate nutrition to the retina. The retina, the most internal tunic, is a thin transparent film (about $100 \mu \mathrm{m}$ in thickness) formed by layers of nerve cells, including the photoreceptors (cone and rod cells) responsible for the vision. ${ }^{10}$ The region of maximum visual acuity is called fovea, and has a diameter of about $1.5 \mathrm{~mm}$. Different types of tissues occupy the inner part of the bulb: the aqueous humor, the crystalline lens and the vitreous body. The aqueous humor is a transparent and colorless liquid located between the cornea and the lens. It is one of the dioptric means of the eye, and has nutritional functions for the avascular ocular tissues of cornea and crystalline lens. The crystalline lens is an avascular organ with the shape of a biconvex lens of about $10 \mathrm{~mm}$ in diameter. It is located at an average distance of $5.4 \mathrm{~mm}$ from the anterior surface of the cornea, and it is able to adapt its refractive power in function of the distance of the object, by modifying its radius of curvature. Finally, the space between the posterior part of the lens and the retina is occupied by the vitreous body, which looks like a clear and transparent gel with an hydration degree close to $98 \% .^{11}$ Some parameters of the human eye are reported in the Appendix Table 1.

\section{Eye phantoms}

Regardless of the area of use, the following characteristics have proved to be

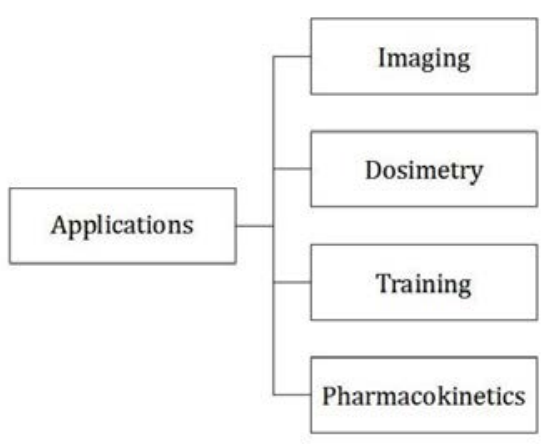

Figure 1. Classification of phantoms as function of their area of application. 
essential in the design of eye phantoms: i) anthropomorphic structure, even simplified, which could be approximated by an axially symmetrical geometry consisting of concentric shells; ii) stability of materials, both in time (conservation techniques and possibility of reuse), and in operating conditions (e.g., temperature sensitivity); iii) low cost of manufacture.

The eye phantoms commercially available or described in literature are listed below, subdivided according to their area of application (i.e. imaging, dosimetry, surgical training, pharmacokinetics).

\section{Phantoms for imaging}

The term medical imaging indicates a branch of medicine and engineering that studies and uses technologies and processes to create a virtual representation, two- or three-dimensional, of a part of an organism, not visible from the outside, for the clinical analysis and the planning of an operation. For instance, radiography and the positrons emission tomography fall in this area. In ophthalmology, particular importance is given to the optical coherence tomography and the ocular ultrasonography.

\section{Phantom for optical coherence tomography}

The optical coherence tomography (OCT) is a non-invasive diagnostic examination which allows to obtain high-resolution scans $(7-10 \mu \mathrm{m})$ of the cornea and retina. OCT is based on a low-coherence interferometric technique, that typically employs near-infrared light (with a wavelength from $700 \mathrm{~nm}$ to $1 \mu \mathrm{m}$ ), allowing a wide penetration depth into biological tissues: a near infrared $(820 \mathrm{~nm})$ beam, generated by a superluminescent diode (or a femtosecond laser), is irradiated onto the retina. The echo propagation times of the light reflected by the retina and by a reference mirror placed at a known distance are compared. The tomograms of an entire section (B-scan) are displayed in real time, using a false color scale that represents the degree of reflectivity of the tissues located at different depth. ${ }^{12-16}$ From a physical point of view, the parameters that allow emulating the eye behavior are: i) the refractive index (dimensionless), i.e. the ratio between the speed of electromagnetic radiation in the vacuum and the speed within a material; ii) the reflectance (dimensionless), i.e. the ratio between the intensity of the reflected and incident electromagnetic waves; iii) the attenuation coefficient $\mathrm{k}_{\lambda}$ (measured in $\mathrm{m}^{-1}$ ), which indicates the reduction of the electromagnetic wave intensity (I), usually described with a mono-exponential fitting in accordance with the Lambert-Beer law (Eq. 1):

$$
\frac{I}{I_{0}}=e^{-k_{\lambda} x}
$$

These quantities depend both on frequency of the electromagnetic wave and the materials through which the radiation is travelling (Figure 2).

Being a relatively new technology, the development of OCT systems strongly depends on the development of phantoms. Several studies have shown that a solid matrix incorporating nano- or micro-particles, with the role of scatter and absorbent, quite closely emulates the eye behavior. The concentration of the scatter particles is based on the speckle pattern, i.e. the spotted pattern typically produced by any coherent imaging tool. 14 Polydimethylsiloxane is one most used materials for fabricating the matrix. It is a silicone rubber characterized by a refractive index of about 1.4 at a wavelength of $1.3 \mu \mathrm{m}$, with an absorption coefficient lower than $0.5 \mathrm{~mm}^{-1}$. Microspheres of titanium dioxide, silica, alumina, and gold nanoparticles are reported as possible scatters, whereas the carbon black can be used as absorbent material. The non-anthropomorphic phantom preparation is relatively simple: the most important steps are the ultrasonic mixing and degassing under vacuum, to obtain a homogeneous distribution of the diffusive and absorbent agents into silicone, without aggregates, sedimentations or air bubbles. These phantoms present stable optical properties for at least one year after their fabrication.

Since inorganic particles present a refractive index higher than biological structures, and given the silicone incompatibility with organic molecules, also biopoly- mers such as fibrin have been tested as matrix. The fibrin hydrogel is usually prepared by dissolving fibrinogen and thrombin powders in a saline solution, and then mixed to form a gel; the preparation time can be reduced by adding calcium chloride. Optical diffusion properties can be obtained by adding tissues constituents, such as blood and lipids. 15

Anthropomorphic phantoms for OCT was produced starting from ocular phantom for ophthalmoscopy (like the OEMI-7 model, Ocular Instruments, Inc., Bellevue, WA), by replacing the retina with an epoxy resin matrix loaded with gold nanoparticles. This phantom was used for three-dimensional characterization of the Point Spread Function (PSF) of an OCT system, i.e. the function that describes how an imaging tool acquires a point source. 16

Altogether, phantoms for OCT do not present fine details of the eye geometry, e.g. the choroidal vascular network, but are more focused on the calibration and eventually on the comparison of different devices.

\section{Phantom for ocular ultrasonography}

Ocular ultrasonography is a non-invasive examination, which, taking advantage of ultrasounds propagation and reflection, enables the study of the internal structures of the eye, by displaying them in twodimensional anatomical sections (B-scan mode). This exam is used to study both the intra-bulbar ocular structures, when direct exploration is prevented by the opacity of the cornea, crystalline lens, and/or vitreous, and diseases, such as tumors, retinal and choroid detachment, malformations and degenerative pathologies of the retina and choroid. ${ }^{17,18}$ The ocular ultrasonography is performed by placing a probe, connected to

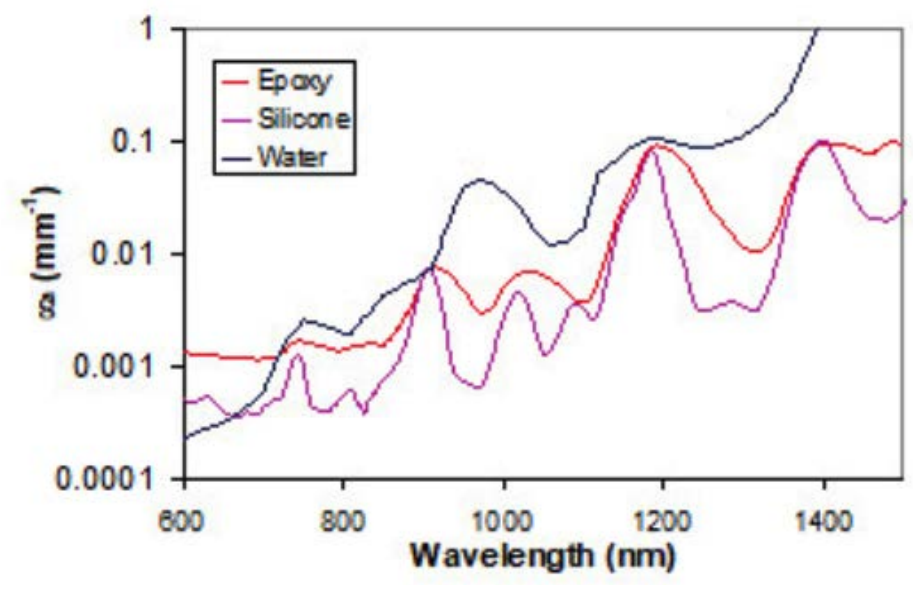

Figure 2. Absorption spectrum of an epoxy resin, a silicone-based material and water. Reproduced with permission of The Optical Society (OSA) from Agrawal A, Connors M, Beylin A, et al. Characterizing the point spread function of retinal OCT devices with a model eye-based phantom. Biomed Opt Express 2012;3:1116-26.16 
the echographer, on the patient's eyelid, after the application of a gel to improve the ultrasonic signal passage. Ultrasound waves are reflected by the tissues, generating return echoes that are picked up and transduced into images. The frequencies of ultrasounds used in ophthalmology range from 8 to $65 \mathrm{MHz}$ : the higher is the frequency, the smaller is the depth into tissues at which the examination can be conducted (Table 1).

Ultrasounds are elastic waves (pressure waves), which propagate inside of a medium with a speed $\mathrm{v}$ that depends on the mechanical properties and density of the medium. In the case of an elastic and isotropic solid, the elastic waves have both a traversal ( $\mathrm{v}_{\mathrm{T}}$, Eq. 2) and a longitudinal speed $\left(v_{L}\right.$, Eq. 3):

$$
\begin{aligned}
& v_{T}=\sqrt{\frac{E}{2 \rho(1+v)}} \\
& v_{L}=\sqrt{\frac{E(1-v)}{\rho(1-2 v)(1+v)}}
\end{aligned}
$$

where $\mathrm{E}[\mathrm{Pa}]$ is the elastic modulus of the material, $v$ (dimensionless) the Poisson's ratio and $\rho\left[\mathrm{kg} \cdot \mathrm{m}^{-3}\right]$ its density. In case of liquids, it will have only the longitudinal components, that is (Eq. 4):

$$
v_{L}=\sqrt{\frac{1}{\rho \beta}}
$$

where $\beta$ is the coefficient of adiabatic compressibility of the medium (the analogue of the elastic modulus for solids) defined as (Eq. 5):

$$
\beta=\left.\frac{\Delta V}{V} \frac{1}{\Delta P}\right|_{Q=0}
$$

where $\mathrm{V}$ indicates the considered volume and $\mathrm{P}$ the pressure at which this volume is subjected to (with an exchange of heat Q equal to zero). Because of the viscoelastic nature of the materials, the intensity of the acoustic waves is attenuated typically following an exponential decreasing pattern, characterized by the coefficient of acoustic attenuation of a substance $(\alpha)$. $\alpha$ typically increases with the frequency, thereby decreasing the depth of penetration of the waves. Acoustic properties of some of the constituents of the human eye are reported in the Appendix Table 2.

When an acoustic wave hits a surface that divides two different substances, it is partly transmitted and partly reflected. The amplitude coefficient of the reflected wave (R) depends on the acoustic impedance of the medium that contains the incident wave and the reflected wave $\left(Z_{1}\right)$ and the acoustic impedance of the medium where the transmitted wave will travel $\left(\mathrm{Z}_{2}\right)$, according to the relation (Eq. 6):

$$
R=\frac{Z_{2}-Z_{1}}{Z_{2}+Z_{1}}
$$

where the acoustic impedance is defined as $\mathrm{Z}=\rho \mathrm{v}$, and it is measured in rayl (1 rayl $\left.=1 \mathrm{~kg} \cdot \mathrm{m}^{-2} \cdot \mathrm{s}^{-1}\right)$. The echography relies on the reflected wave, which reveals all the interfaces that determine variations of the acoustic impedance. From the above considerations, a phantom for ocular ultrasonography should mimic, as much as possible, the mechanical properties of the eye, which can be summarized in the impedance and attenuation parameters (Table 2).

Considering that nowadays about $2 \%$ of the admissions to the emergency room concerns trauma and infections to the visual apparatus, the ocular ultrasonography is becoming a fast-diagnostic tool and therefore the request of phantoms comes more from teaching needs than from the calibration of instruments. An ocular phantom capable to recreate pathophysiological situations, with a cost of about $\$ 20$, can be manufactured using readily available materials. The eye is simulated by a solution of gelatin mixed to psyllium, using as a container a plastic ball, whereas the crystalline lens is reproduced by an elliptical incision on the sphere itself. Once the model is obtained, the pathological condition of hemorrhage is simulated by injecting a hyperechoic fluid, while to recreate the retinal detachment the back of the ocular phantom is carved. If frozen, the phantom can be reused, although the gelatin with psyllium loses its properties within few weeks. ${ }^{19}$

\section{Phantoms for dosimetry}

Dosimetry is a branch of physics that deals with the calculation and measurement of the amount of energy absorbed by a material when subjected to an electromagnetic field.

The frequency $(v)$ is the fundamental characteristic that distinguishes the various electromagnetic fields and determines their properties; it represents the number of oscillations made by the wave in one second (unit time), measured in Hertz (Hz). Frequency is closely related with the wavelength $(\lambda,[\mathrm{m}])$, which is the distance traveled by the wave during a period and corresponds to the distance between two maximums or two minimums of the wave, through the relation (Eq. 7):

$$
v=\frac{c}{\lambda}
$$

where $\mathrm{c}$ is the speed of light $\left(3 \cdot 10^{8} \mathrm{~m} / \mathrm{s}\right)$. The energy of a radiation (U, expressed in Joules $[\mathrm{J}]$ ) is directly proportional to the frequency (Eq. 8):

$U=h \cdot v$

Eq. 8

where $\mathrm{h}=6.626 \cdot 10^{-34} \mathrm{~J} \times \mathrm{s}$ is the Planck's constant.

The effects of electromagnetic radiations on living organisms depend principally on two factors: i) the frequency of the radiation, i.e. the type, which determines the energy carried by the radiation itself; ii) the exposure mode, i.e. the intensity of the radiation and the duration of exposure,

Table 1. Frequency, resolution and depth of penetration used in ocular ultrasound.

\begin{tabular}{lcc} 
Frequency [MHz] & Resolution [jum] & Depth of penetration [mm] \\
$8-10$ & $200-500$ & $30-40$ \\
$35-50$ & $20-60$ & $5-8$ \\
\hline 65 & 5 & 1 \\
\hline
\end{tabular}

Table 2. Possible materials for the construction of anthropomorphic ocular phantoms for surgical training.

\begin{tabular}{ll} 
Eyye area & Material \\
$\begin{array}{ll}\text { Sclera } \\
\text { Choroid }\end{array}$ & $5 \%(\mathrm{w} / \mathrm{v})$ polycaprolactone in chloroform \\
\hline Retina & Polydimethylsiloxane \\
Vitreous humor & $\begin{array}{l}4 \%(\mathrm{w} / \mathrm{v}) \text { of gelatin in deionized water crosslinked with } 0.2 \%(\mathrm{w} / \mathrm{v}) \text { of genipin } \\
31.5 \%(\mathrm{w} / \mathrm{v}) \text { of polyvinyl alcohol in deionized water }+2.5 \%(\mathrm{w} / \mathrm{v}) \\
\text { of gelatin in deionized water }\end{array}$
\end{tabular}


which determines the energy deposited by the radiation, and the exposed parts of the body.

Regarding the frequency of the radiation, it is common to distinguish between: i) ionizing radiations, with a frequency high enough to be able to ionize the atoms of the exposed substance, including X-rays and gamma rays. They have very high frequency $(>3000 \mathrm{THz})$ and are able to directly ionize matter, whatever is their intensity. Ionizing radiations can modify molecular structures, including DNA, producing longterm biological effects; ii) non-ionizing radiations, characterized by a frequency lower than $3000 \mathrm{THz}$, are not able to produce ionization in the exposed materials, and their biological effects are related to the induced electric currents and the heating effect connected to them.

Given the different modalities of interaction with materials, the dosimetry for the two types of radiation requires different types of phantom. In any case, just for the definition of phantom proposed in this work, the biological effects cannot be simulated, since no living material is employed. 20

\section{Phantoms for non-ionizing radiations}

The International Commission on NonIonizing Radiation Protection represents the reference concerning safety of exposure to electromagnetic fields; it is officially recognized by the World Health Organization and the International Agency for Cancer Research. In the frequency spectrum of non-ionizing radiations, the high-frequency field, from $100 \mathrm{kHz}$ to $300 \mathrm{GHz}$, are widely used by many technological devices (from Wi-Fi networks to GSM network). The Specific Absorption Rate (SAR), that is the specific power absorbed per mass unit $[\mathrm{W} / \mathrm{kg}]$, is widely accepted as the most relevant quantity to evaluate the biological effects of these radiations. The calculated value is greatly affected by the geometry of the body part exposed to radiofrequency waves, as well as the exact location and geometry of the source. For this reason, tests should be performed with every specific source (such as a particular model of a mobile phone), and in the exact position in which it will be used under operating conditions (i.e., in this specific case, next to the ear). The SAR is therefore measured in the maximum absorption conditions by the human body: a lower value of SAR corresponds a lower tissues heating and, consequently, a reduced potential risk to health. With regards to the eye, the SAR limit for an average tissue of $10 \mathrm{~g}$ is $2 \mathrm{~W} / \mathrm{kg}$ over a frequencies range between $0.5 \mathrm{GHz}$ and 3.5 $\mathrm{GHz}$, whereas the maximum temperature increase should not exceed $1^{\circ} \mathrm{C}$.
In the case of an ocular phantom, the basic parameters to be reproduced, in addition to the geometric dimensions, are: i) the relative permittivity (dimensionless), which explains the behavior of a dielectric material in the presence of an electric field; it is typically a complex function of the frequency; ii) the conductivity $[\mathrm{S} / \mathrm{m}]$, which relates the electric current density with the electric field intensity.

Generally, the SAR is estimated by coupling a virtual phantom for calculation (analytical or semi-analytical) with the experiments carried out on a physical phantom, characterized by stylized anthropomorphic features. ${ }^{21}$

The computational model, which is implemented in several numerical simulation software, allows coupling the Maxwell equations for electromagnetism with heat transfer equation (Eq. 9):

$$
\nabla \cdot(k \nabla T)+\rho S A R+A-B\left(T-T_{b}\right)=C \rho \frac{\partial T}{\partial t}
$$

where $\mathrm{T}$ is the temperature $[\mathrm{K}], \mathrm{k}$ is the thermal conductivity $\left[\mathrm{W} \cdot \mathrm{m}^{-1} \cdot \mathrm{K}^{-1}\right], \mathrm{C}$ is the spetransport associated to the bloodstream $\left[\mathrm{W} \cdot \mathrm{m}^{-3} \cdot \mathrm{K}^{-1}\right]$, and $\mathrm{A}$ represents the basal metabolic rate $\left[\mathrm{W} \cdot \mathrm{m}^{-3}\right]$.

From an experimental point of view, it has been observed that liquid phantoms, consisting of solutions of water, sucrose and sodium chloride, reproduce, for certain wavelengths, the behavior of the eye as a whole (Table 3).

The geometry of these phantoms is generally kept as simple as possible. Furthermore, the presence of vascularized tissues that can modify the temperature profile is not recreated, indirectly simulating the worst case.

\section{Phantom for ionizing radiations}

Radioprotection, which is the branch of science that deals with the protection of humans from the harmful effects of ionizing radiations, is based on three fundamental principles established by the International Commission on Radiological Protection (ICRP): justification, optimization and cific heat $\left[\mathrm{J} \cdot \mathrm{kg}^{-1} \cdot \mathrm{K}^{-1}\right], \mathrm{B}$ weighs the heat

doses limitation. The amount of radiation absorbed by an organism can be measured as: i) absorbed dose, i.e. the energy that the radiation yields to the material per unit mass (expressed in $[\mathrm{J} / \mathrm{kg}]$ or gray [Gy]); ii) equivalent dose, i.e. the energy that the radiation yields to the material per unit mass, and weighted for an appropriate coefficient which depends on the type of radiation (measured in Sievert, [Sv]); iii) effective dose, which, besides the equivalent dose, takes into account, through an appropriate coefficient, the type of tissue involved (measured in Sievert as well).

Considered the relevance both in terms of applications (such as computed axial tomography) $)^{22}$ and dangerousness, phantoms for ionizing radiations dosimetry are of great importance. There are several properties that can be used to determine the goodness of a material to emulate a biological tissue: the density, the effective atomic number, the electronic density. The coefficient of absorption per unit mass is the most commonly accepted parameter to indicate how much energy is locally deposited in the tissue of interest. Moreover, it should be considered that these parameters greatly depend on the type of radiation.

A phantom for ionizing radiation dosimetry is typically not-anthropomorphic (or grossly reproduces some internal organs) and it is composed of liquid, jelliform or waxy materials. In this way dosimeters (ionization chambers, thermoluminescent dosimeters, radiographic films, etc.) can be easily accommodated to quantify the dose in the interior parts of the phantom. ${ }^{23}$

In the ionizing radiation field, virtual phantoms found extensive use: currently, the ICRP has appointed two virtual phantoms (a man and a woman) as reference, but several others are described in literature. Most of these models are based on Monte Carlo-type codes. The Monte Carlo method is part of the family of non-parametric statistical methods, and it is used to derive estimates through simulations of a physical phenomenon, in which the variables are too numerous and becomes difficult, if not impossible, to perform analytical calculations. In these cases, it is appropriate to use simulation methods, in which the mechanism under examination is reproduced by

Table 3. Composition of the liquid phantom for Specific Absorption Rate measurements at a frequency of $1.8 \mathrm{GHz}$.

\begin{tabular}{lccccc} 
Section & \multicolumn{3}{c}{ Material [\%] } & $\varepsilon_{\mathrm{r}}$ & $\sigma[\mathrm{S} / \mathrm{m}]$ \\
Eye & Water & Sucrose & Sodium chloride & 69 & 1.9 \\
Other tissues & 89 & 10 & 1 & & 0.2 \\
\hline
\end{tabular}


replacing the analytical evaluation with empirical observations of the studied event. The frequency, with which the simulated event is observed, constitutes an assessment of probability. The simulation calculates a series of possible evolution of the phenomenon under consideration, trying to explore, as detailed as possible, the whole space of the parameters of the phenomenon. After calculating this random sample, the simulation performs measurements of the quantities of interest of that sample. ${ }^{24,25}$ In general, this method can be applied to any phenomenon in which the probability of occurrence is known. The larger is the statistical sample, the lower is the uncertainty on the solution.

In the field of ophthalmology, virtual dosimetry models are applied to analyze the radiations dose involved in a process of brachytherapy for the treatment of ocular melanoma. ${ }^{26}$ The brachytherapy is a form of radiotherapy where a source of radiation is placed inside or near the area to be treated. In the case of ocular melanoma, the radioactive plaque is sutured to the sclera in correspondence of the base of the tumor and left in place for the time necessary to emit the required dose (usually 4-7 days). By analyzing different sources, on the basis of the radionuclides and their distribution, the combination which optimizes the distribution of emitted radiations can be identified. ${ }^{27,28}$ Other applications can be found in the planning of the proton therapy. ${ }^{29}$

Physical phantoms for dosimetry are thus generally used for the validation of a virtual phantom for calculations, which find extensive application in telecommunication research (e.g. design of antennas for smartphones), and in medical industry (new products for diagnosis and treatments based on ionizing radiation).

\section{Phantoms for surgical training}

The modern surgery not only seeks to find a remedy for the patient's pathology, but at the same time tries to minimize the discomforts due to the operation and maximize the therapeutic success; thanks to the endoscopic approach, surgery is evolving toward a less invasive discipline, which tends to limit the trauma of the intervention itself.

In the field of ophthalmology, the minimally-invasive vitreoretinal surgery procedure was introduced in 2002. It is known as Transconjunctival Sutureless Vitrectomy (TSV) and foresees the use of $23-\mathrm{G}$ or $25-\mathrm{G}$ trocars (microcannules) that allow the creation of channels through which the surgical instruments necessary for the operation are introduced into the vitreous body. Since the outer diameter of these microcannules is equal to 0.6 or $0.5 \mathrm{~mm}$ (23-G or $25-\mathrm{G}$, respectively), the incisions left on the sclera are so small that can be sealed without sutures. The procedure does not cause any surgical trauma to the conjunctiva, does not require suturing to the sclera, and ensures extremely quick recovery times. 30

The endoscopic approach is also applied in computer-assisted surgery (CAS): the Da Vinci robot is the most famous example, and it is by now used in clinical practice, whereas there are ongoing researches to perform also TSV with the CAS approach.

Regardless of the presence of the computer, the endoscopic surgery compels the surgeon to operate with a two-dimensional view that flattens the depth of the operative field, with a type of instrumentation that eliminates the tactile sensation characteristic of the surgical gesture and reverses the direction of the movements because of the presence of the fulcrum represented by the access point of the trocar. It has been scientifically proved that these limitations can be overcome through a training activity which involves the use of physical or virtual phantoms. These brought the intraoperative complications associated with minimallyinvasive surgery to be equivalent in percentage to those of traditional surgery.

It is easy to understand that a phantom for surgical training, but also for testing the instruments for the TSV, has to reproduce the mechanical properties of the eye.

The elasticity of a material is often described by the elastic modulus $\mathrm{E}[\mathrm{Pa}]$, which for linear elastic materials is defined as the initial slope of the stress-strain curve (Table 1). Many biological materials, including the ocular tissues, have a non-linear behavior when deformation exceeds 10\%.31-33 The biological tissues actually present a viscoelastic type behavior, with characteristics in common both with viscous and elastic materials, showing phenomena of hysteresis, creep and stress relaxation.

The viscoelastic behavior can be described using the standard linear solid model, consisting of an elastic element in parallel to a branch composed by an elastic and a viscous element: in the specific case of the cornea, for example, the elastic elements are about $8.5 \mathrm{MPa}$, while the viscous element has a viscosity (that is the resistance of a fluid to the flow) of about 16.5 $\mathrm{kPa} \cdot \mathrm{s} .34$

This analysis can be only partially applied to the vitreous humor, which is a delicate transparent collagenous gel that fills the posterior chamber of the eye. It weighs about $4 \mathrm{~g}$ and represents approximately two-thirds of the ocular volume. The vitreous body is delimited by various structures: posterolaterally it is in contact and adheres to the retina and anteriorly with the crystalline lens and other structures. It is composed of water $(>98 \%)$, it is avascular and virtually acellular and for these reasons it has been historically considered as a simple inert filler. However, in the last decades it has become clear that the vitreous plays an essential role in supporting the vision and in the development of disorders.

From a structural point of view, the vitreous humor derives its properties from its hydrated double network composed of type II collagen fibers and high molecular weight hyaluronic acid macromolecules. The lubricating properties of its molecules and its fragile structure make rheological measurements (bulk measurements, magnetic micro-rheology, tracking in vivo on humans during vision tasks, acoustic techniques) difficult to be performed. The viscosity of the vitreous humor is strongly dependent on the experimental procedures: if approximated to a Newtonian fluid, its value is $4 \mathrm{~Pa} \cdot \mathrm{s} .{ }^{35}$

A list of possible materials which can be used for the construction of an anthropomorphic phantom is provided in Table 2.36 For the vitreous humor, in particular, in addition to those listed in the table, several types of gels have been tested, such as polyacrylamide and polyvinylpyrrolidone, that have been used experimentally also as substitutes in vivo, however with unsatisfactory results. ${ }^{37}$

Anthropomorphic phantoms for applications related to training and to testing of surgical instruments present an anatomical shape, or an hemispherical one, in the case that the interest is focused only on the anterior or posterior part of the eye. ${ }^{36}$ These are usually produced by assembling layer by layer the various components, within a rigid shell that represents the bone cavity. This latter can be stylized or reproduce anthropomorphic features. ${ }^{36,38}$ The close collaboration between expert surgeons and bioengineers is however fundamental for the development of this type of phantom, whose ultimate goal is to reproduce the same feeling experienced during the surgical procedures.

\section{Phantoms for pharmacokinetics}

The pharmacokinetics is a branch of pharmacology that quantitatively studies the absorption, the distribution, the metabolism and the elimination of drugs. In particular, it takes into account the effects of the organism on the drug, that is the mechanisms that allow to reach or to maintain specific concentrations of drugs in the various 
Table 4. Classification of some of the eye phantoms presented in this review according to their construction features.

\begin{tabular}{|c|c|c|}
\hline & Non-anthropomorphic & Anthropomorphic \\
\hline Physical & $\begin{array}{l}\text { Film and blocks for OCT } \\
\text { development,15 chambers } \\
\text { for pharmacokinetic }{ }^{39}\end{array}$ & $\begin{array}{l}\text { Phantoms for ultrasonography } 19 \\
\text { and for retinal surgery tools } \\
\text { development } 36,38\end{array}$ \\
\hline Virtual & $\begin{array}{l}\text { Computational models with } \\
\text { a spherical geometry for } \\
\text { brachytherapy27 }\end{array}$ & $\begin{array}{l}\text { Anatomical eye phantoms for } \\
\text { non-ionizing21 and ionizing } \\
\text { dosimetry28,29 }\end{array}$ \\
\hline
\end{tabular}

compartments of the organisms. ${ }^{39,40}$

The diffusion of drugs within the vitreous humor after an injection is interesting in the clinical setting. Since this is an avascular tissue, the diffusion of the drug, injected with concentration $\mathrm{C}$, can be described by Fick's law (Eq. 10):

$$
\frac{\partial C}{\partial t}=D \nabla^{2} C
$$

where $\mathrm{D}$ is the diffusivity $\left[\mathrm{m}^{2} / \mathrm{s}\right]$ (typically

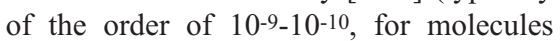
with a molecular weight of some $\mathrm{kDa}$ ). Once injected and diffused, the drug is metabolized, decreasing with a certain elimination rate, typically approximated with a kinetic of the first order.

Phantom for the pharmacokinetics, already commercialized for other types of tissues, such as the cerebral tissue, are instead at the initial stage in ophthalmology, with only a few examples in literature. For instance, a phantom with two compartments (one for the gel, which mimics the vitreous humor, and the other for sampling) separated by a membrane, has been used to study the behavior of a drug in a substitute of vitreous humor based on polyvinyl alcohol. 36,39

\section{Discussion}

The development of phantoms requires a deep knowledge of fundamental and applied physics, ${ }^{14,25}$ chemistry and material science, ${ }^{31-34}$ as well as expertise in biology 39,40 and of course in medicine. 19,30 Collaboration between biomedical engineers and physicians is instrumental for clearly identifying not only the clinical need, 19 but also the level of details required for simulating the surgical procedures. ${ }^{36}$

Excluding the development costs, phantoms are generally not expensive, 19 especially if the savings in terms of design surgical error reductions are considered. For this reason, conservation and reusability of phantoms can be considered a problem more from a practical than an economic
Table 4 organizes some of the eye phantoms presented in this review according to their construction features, and shows that for the same application (e.g., dosimetry) different types of phantoms are available. The use of the same phantom, as it is, for different application is instead not recommended.

Theoretical and experimental researches are critical for the development of new materials able to mimic the behavior of living tissues respect to specific physical or chemical phenomena. With the introduction of Additive Manufacturing technologies, applied to rapid prototyping and rapid tooling, the difficulties in phantoms fabrication (even for anthropomorphic one) is a minor issue. In this sense, efforts should be put in identifying the most suitable fabrication technology and eventually modify the desired material to be manufactured in the final product.

As regards virtual phantoms, processing power is no more a limit for computational models, given the advances in Information and Communications Technology tools as well as the limited complexity and geometrical size of the eye, compared to other regions of the human body. Also in this field, the theoretical development of equations describing the (bio-)material behavior constitutes the most promising field of research.

\section{Conclusions}

The present review has proposed a systematic approach for classifying phantoms used in medicine and bioengineering, which have been used for describing phantoms for ophthalmology.

The review highlighted that there is no ideal ocular phantom which can be used indifferently for any type of measure or surgical operation: some of the desired properties are often obtained at the expense of others (e.g., optical properties vs mechanical properties). Therefore, the best solution seems to be the use of different phantoms according to their specific field of applica- point of view. tion. Fabrication and computational technologies are mature for supporting the development of new phantoms, with desired complexity, while most of the effort should be put in the development of suitable materials able to reproduce the desired behavior of the human eye. From this point of view, experimental research has to be framed by a theoretical description, which can be implemented as mathematical equations in in-silico models.

\section{References}

1. Glasser O. WC Roentgen and the discovery of the Roentgen rays. Am J Roentgenol 1995;165:1033-40.

2. Kneebone R. Simulation in surgical training: educational issues and practical implications. Med Educ 2003;37: 267-77.

3. Petzold R, Zeilhofer HF, Kalender WA. Rapid prototyping technology in medicine - basics and applications. Comput Med Imag Grap 1999;23:277-84.

4. O'Connor MD. The 3R principle: advancing clinical application of human pluripotent stem cells. Stem Cell Res Ther 2013;4:21.

5. Sommer FG, Filly RA, Edmonds PD, et al. A phantom for imaging biological fluids by ultrasound and CT scanning. Ultrasound Med Biol 1980;6:135-40.

6. Ferrari P, Gualdrini G. An improved MCNP version of the NORMAN voxel phantom for dosimetry studies. Phys Med Biol 2005;50:4299.

7. Lafon C, Zderic V, Noble ML, et al. Gel phantom for use in high-intensity focused ultrasound dosimetry. Ultrasound Med Biol 2005;31:1383-9.

8. Fornage BD. A simple phantom for training in ultrasound-guided needle biopsy using the freehand technique. J Ultras Med 1989;8:701-3.

9. Thaker NG, Kudchadker RJ, Swanson $\mathrm{DA}$, et al. Establishing high-quality prostate brachytherapy using a phantom simulator training program. Int J Radiat Oncol 2014;90:579-86.

10. Csillag A. Atlas of the sensory organs: functional and clinical anatomy. New York: Humana Press; 2005.

11. Oyster CW. The human eye. Sunderland, MA: Sinauer; 1999.

12. Baxi J, Calhoun WR, Sepah YJ, et al. Retina-simulating phantom for optical coherence tomography. J Biomed Opt 2013;19:21106.

13. Corcoran A, Muyo G, van Hemert J, et al. Application of a wide-field phantom eye for optical coherence tomography and reflectance imaging. J Mod Optic 
2015;62:1828-38.

14. Lamouche G, Kennedy BF, Kennedy $\mathrm{KM}$, et al. Review of tissue simulating phantoms with controllable optical, mechanical and structural properties for use in optical coherence tomography. Biomed Opt Express 2012;3:1381-98.

15. Kennedy F, Loitsch S, McLaughlin RA, et al. Fibrin phantom for use in optical coherence tomography. J Biomed Opt 2010;15:030507.

16. Agrawal A, Connors M, Beylin A, et al. Characterizing the point spread function of retinal OCT devices with a model eye-based phantom. Biomed Opt Express 2012;3:1116-26.

17. Dastevska-Djosevska E. Ultrasonography in ocular trauma. Hypothesis 2013;12:13.

18. Vrablik ME, Snead GR, Minnigan HJ, et al. The diagnostic accuracy of bedside ocular ultrasonography for the diagnosis of retinal detachment: a systematic review and meta-analysis. Ann Emerg Med 2015;65:199-203.

19. Jafri F, Runde D, Saul T, Lewiss RE. An inexpensive and easy simulation model of ocular ultrasound that mimics normal anatomy as well as abnormal ophtalmologic conditions. J Ultras Med 2011;30:569-73.

20. Podgorsak EB. Review of radiation oncology physics: a handbook for teachers and students. Vienna, Austria: IAE Agency; 2003.

21. Liu L, Nikolova NK, Sangary NT. Feasible methods for the evaluation of the Specific Absorption Rate and the temperature rise in the human eyes. Microwave Symposium Digest 2009;1321-4.
22. Hopper KD, Neuman JD, King SH, Kunselman AR. Radioprotection to the eye during CT scanning. Am J Neuroradiol 2001;22:1194-8.

23. DeWerd LA, Kissick M. The phantoms of medical and health physics - Devices for research and development. New York: Springer Science + Business Media; 2014

24. Hastings WK. Monte Carlo sampling methods using Markov chains and their applications. Biometrika 1970;57:97109.

25. Lesperance M, Martinov M, Thomson RM. Monte Carlo dosimetry for 103Pd, 125I, and $131 \mathrm{Cs}$ ocular brachytherapy with various plaque models using an eye phantom. Med Phys 2014;41:031706.

26. Devlin PM. Brachytherapy: applications and techniques. Philadelphia: Lippincott Williams \& Williams; 2007.

27. Gagne NL, Rivard MJ. COMS eye plaque brachytherapy dosimetric sensitivity to source photon energy and seed design. Appl Radiat Isot 2013;79:62-6.

28. Lesperance $M$, Inglis-Whalen $M$, Thomson RM. Model-based dose calculations for COMS eye plaque brachytherapy using an anatomically realistic eye phantom. Med Phys 2014;41:021717.

29. Alghamdi A, Ma A, Marouli M, et al. A high-resolution anthropomorphic voxel-based tomographic phantom for proton therapy of the eye. Phys Med Biol 2007;52:51-9.

30. Rizzo S, Patelli F, Chow DR. Vitreoretinal Surgery. Berlin-Heidelberg: Springer-Verlag; 2009.

31. Eilaghi A, Flanagan JG, Tertinegg I, et al. Biaxial mechanical testing of human sclera. J Biomech 2010;43:1696-701.

32. Friberg TR, Lace JW. A comparison of the elastic properties of human sclera. Exp Eye Res 1988;47:429-36.

33. Jones IL, Warner M, Stevens JD. Mathematical modelling of the elastic properties of retina: a determination of Young's modulus. Eye London Engl 1992;6:556-9.

34. Glass DH, Roberts CJ, Litsky AS, Weber PA. A Viscoelastic biomechanical model of the cornea describing the effect of viscosity and elasticity on hysteresis. Invest Ophthalmol Vis Sci 2007;49:3919-26.

35. Tokita M, Fujiya Y, Hikichi K. Dynamic viscoelasticity of bovine vitreous body. Biorheology 1984;21:751-6.

36. Fogli G, Orsi G, De Maria C, et al. New eye phantom for ophthalmic surgery. J Biomed Opt 2014;19:6-8.

37. Negron LA, Viola F, Black EP, et al. Development and characterization of a vitreous mimicking material for radiation force imaging. IEEE Trans Ultrason Ferroelectr Freq Control 2002;49:1543-51.

38. Richa R, Balicki M, Sznitman R, et al. Vision-based proximity detection in retinal surgery. IEEE Trans Biomed Eng 2012;59:2291-301.

39. Rescigno A. Foundations of pharmacokinetics. New York: Kluver Academy/Plenum Publishers; 2003.

40. Labaune JP. Handbook of pharmacokinetics: toxicity assessment of chemicals. Chichester: Ellis Horwood; 1989. 